details involved in the process remain incompletely understood (18-21). An interesting question stemming from the work of Vicent et al. (3) is whether their observations regarding senescence also occur in vivo. The authors show that deletion of Wt1 in the context of oncogenic Kras in the lung results in a significant reduction in tumor volume but not in the total number of lesions, indicating that Wt1 loss likely affects tumor progression rather than initiation. This fits with a role for $\mathrm{Wt} 1$ in repressing the senescence response to oncogenic Kras as observed in vitro. However, more studies are needed to conclusively prove that Wt 1 controls Krasinduced senescence in vivo.

\section{Acknowledgments}

The work in the Kissil laboratory is supported in part by NIH grant R01CA124495 and the American Cancer Society.

Address correspondence to: Joseph L. Kissil, Molecular and Cellular Oncogenesis Program, The Wistar Institute, Philadel- phia, Pennsylvania 19104, USA. Phone: 215.898.3874; Fax: 215.898.3572; E-mail: jkissil@wistar.org.

1. Karnoub AE, Weinberg RA. Ras oncogenes: split personalities. Nat Rev Mol Cell Biol. 2008;9(7):517-531.

2. Kaelin WG Jr. The concept of synthetic lethality in the context of anticancer therapy. Nat Rev Cancer. 2005;5(9):689-698

3. Vicent $S$, et al. Wilms tumor 1 (WT1) regulates KRAS-driven oncogenesis and senescence in mouse and human models. J Clin Invest. 2010; 120(11):3940-3952.

4. Westbrook TF, Stegmeier F, Elledge SJ. Dissecting cancer pathways and vulnerabilities with RNAi. Cold Spring Harb Symp Quant Biol. 2005;70:435-444.

5. Downward J. Use of RNA interference libraries to investigate oncogenic signalling in mammalian cells. Oncogene. 2004;23(51):8376-8383.

6. Bernards R, Brummelkamp TR, Beijersbergen RL. shRNA libraries and their use in cancer genetics. Nat Methods. 2006;3(9):701-706.

7. SharmaS, Rao A. RNAi screening: tips and techniques. Nat Immunol. 2009;10(8):799-804.

8. Barbie DA, et al. Systematic RNA interference reveals that oncogenic KRAS-driven cancers require TBK1. Nature. 2009;462(7269):108-112.

9. Luo J, et al. A genome-wide RNAi screen identifies multiple synthetic lethal interactions with the Ras oncogene. Cell. 2009;137(5):835-848.

10. Scholl C, et al. Synthetic lethal interaction between oncogenic KRAS dependency and STK33 suppression in human cancer cells. Cell. 2009;137(5):821-834.
11. Hacker H, Karin M. Regulation and function of IKK and IKK-related kinases. Sci STKE. 2006; 2006(357):re13.

12. Rivera MN, Haber DA. Wilms' tumour: connecting tumorigenesis and organ development in the kidney. Nat Rev Cancer. 2005;5(9):699-712.

13. Yang L, Han Y, Suarez Saiz F, Minden MD. A tumor suppressor and oncogene: the WT1 story. Lenkemia. 2007;21(5):868-876.

14. Serrano M, Lin AW, McCurrach ME, Beach D, Lowe SW. Oncogenic ras provokes premature cell senescence associated with accumulation of p53 and p16INK4a. Cell. 1997;88(5):593-602.

15. Tuveson DA, et al. Endogenous oncogenic $\mathrm{K}$-ras(G12D) stimulates proliferation and widespread neoplastic and developmental defects. Cancer Cell. 2004;5(4):375-387.

16. DeNicola GM, Tuveson DA. RAS in cellular transformation and senescence. Eur J Cancer. 2009;45(Suppl 1):211-216.

17. Young NP, Jacks T. Tissue-specific p19Arf regulation dictates the response to oncogenic K-ras. Proc Natl Acad Sci U S A. 2010;107(22):10184-10189.

18. Braig $M$, et al. Oncogene-induced senescence as an initial barrier in lymphoma development. Nature. 2005;436(7051):660-665.

19. Chen Z, et al. Crucial role of p53-dependent cellular senescence in suppression of Pten-deficient tumorigenesis. Nature. 2005;436(7051):725-730.

20. Collado M, et al. Tumour biology: senescence in premalignant tumours. Nature. 2005;436(7051):642.

21. Michaloglou C, et al. BRAFE600-associated senescence-like cell cycle arrest of human naevi. Nature. 2005;436(7051):720-724.

\title{
Are there more tricks in the bag for treating thrombocytopenia?
}

\author{
Andrew D. Leavitt \\ Department of Laboratory Medicine, University of California, San Francisco, San Francisco, California, USA.
}

\begin{abstract}
Thrombocytopenia, an abnormally low number of circulating platelets, results from inadequate platelet production, splenic platelet sequestration, or accelerated platelet clearance. Platelet transfusions are now the cornerstone for treating thrombocytopenia. With an ever-expanding demand for platelets, and with many patients having an inadequate response to platelet transfusions, new strategies are needed to treat thrombocytopenia. In this issue of the JCI, Fuentes et al. present provocative data regarding the use of direct megakaryocyte infusions as a novel approach to manage this vexing clinical problem.
\end{abstract}

It was only 100 years ago that James Wright reported that his modification of the Romanowsky stain could unequivocally identify platelets on a peripheral blood smear (1). Applying his new stain and careful observations to bone marrow specimens, in 1910 Wright proposed that "the blood platelets are detached portions

Conflict of interest: The author has declared that no conflict of interest exists.

Citation for this article: J Clin Invest. 2010; 120(11):3807-3810. doi:10.1172/JCI45179. or fragments of the cytoplasm of the megakaryocytes, which are in such relation to the blood channels in the marrow that detached portions of their cytoplasm are quickly carried by the blood current into the circulation. The breaking up of the cytoplasm into the platelets occurs only in cells which have reached a certain stage of growth and development, and is probably rapidly completed when once begun. It takes place in various ways but usually by the pinching off of small rounded projections or pseudopods from the cell body or from larger pseudopods, or by the segmentation of slender pseudopods, or by the pinching off of longer or shorter pseudopods which may or may not undergo segmentation later" (2).

Data accumulated over the past 10 years strongly suggest that platelets emerge from the tips of "proplatelets," the long cytoplasmic extensions generated by large, mature, polyploid megakaryocytes (Figure 1). Proplatelet extensions are produced by anti-parallel microtubule sliding powered by dynein motors, and repeated branching increases the number of platelet-releasing ends (3). Platelet granules and organelles are manufactured in the megakaryocyte cell body, transported down the extensions by kinesin motors, and then packaged into budding platelets (3). The proplatelet ends uniquely contain a marginal microtubule coil similar to that seen in mature platelets, supporting the idea that platelets are released from proplatelet tips and helping explain the biologi- 


\section{A Megakaryocytopoiesis}

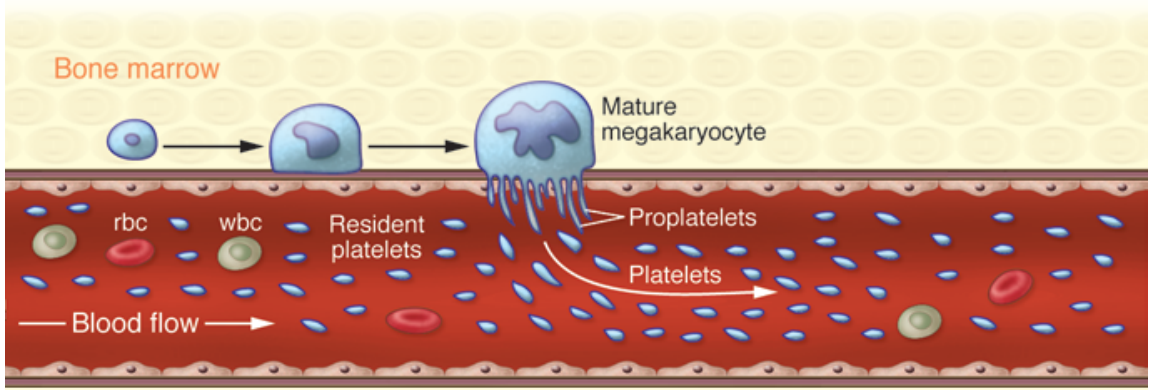

Injection of mature megakaryocytes

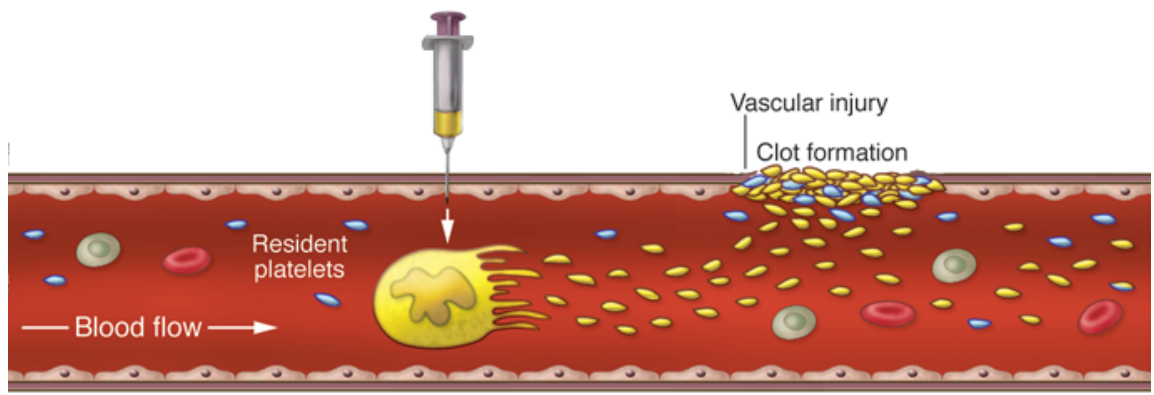

Figure 1

Megakaryocytopoiesis and platelet production. (A) A model for megakaryocytopoiesis demonstrates the progressive increase in size as megakaryocytes mature in the marrow, with mature megakaryocytes developing cytoplasmic projections called proplatelets. Platelet release occurs from the ends of proplatelet projections, with at least some platelet release occurring directly in the marrow circulation from proplatelet projections that extrude through the marrow's fenestrated vasculature. (B) Fuentes et al. (19) have demonstrated that infused mature megakaryocytes release platelets into the circulation, thereby increasing circulating platelet counts, and that these platelets contribute to hemostasis at the site of vascular injury.

cal utility of extensive proplatelet branching. Recent data suggest that individual platelets may retain the ability to "divide" in circulation through mechanisms similar to those involved in proplatelet formation (4).

\section{Thrombocytopenia and thrombopoietin}

Acquired thrombocytopenia develops secondary to inadequate platelet production in the setting of primary or secondary marrow disorders, as well as secondary to platelet sequestration in the spleen and to destruction in the periphery. The latter is typically a result of autoimmune or druginduced immune-mediated processes. Two of the more common clinical settings requiring platelet transfusion therapy include patients with hematologic disorders receiving high-dose chemotherapy or undergoing bone marrow transplantation, and patients with severe liver dis- ease. While splenomegaly contributes to thrombocytopenia in the setting of severe liver disease, inadequate thrombopoietin (TPO) production by the failing liver also contributes significantly.

The 1994 cloning of human TPO (5-7), the primary cytokine required for normal numbers of bone marrow megakaryocytes and circulating platelets, engendered great excitement for the treatment of thrombocytopenia. Two products underwent clinical trials, a full-length recombinant molecule administered intravenously and a pegylated, amino-terminal fragment administered subcutaneously (8). These molecules successfully minimized the extent and duration of thrombocytopenia during chemotherapy for non-hematologic malignancies, including high-dose platinum-based treatment for gynecologic tumors, where the treatment reduced the need for platelet transfusions from $77 \%$ of patients to $24 \%$
(9). However, neither agent meaningfully attenuated the extent or duration of thrombocytopenia when given to patients either receiving high-dose chemotherapy for leukemia or undergoing bone marrow transplantation. Interestingly, TPO treatment of donor mice prior to stem cell collection accelerated platelet reconstitution (10), and TPO stimulation of stem cell donors reduced platelet transfusion requirements in a human trial (11), suggesting that treatment of the cellular transplant product, as opposed to the transplant recipient, could have clinical value.

A single dose of TPO administered to platelet donors was shown to double the platelet count 14 days after injection and increase apheresis platelet yield by nearly 3 -fold (12), a promising finding for improving platelet availability to treat thrombocytopenia. However, excitement for TPO abruptly diminished when 4 clinical trial patients and 13 of 334 healthy subjects in a large safety trial developed antibodies against the administered TPO therapeutic that cross-reacted with native TPO, producing profound and prolonged thrombocytopenia (8). While this complication developed only in patients receiving the subcutaneously administered pegylated, amino-terminal TPO fragment formulation, clinical development was halted for both products.

IL-11 is the only FDA-approved agent to treat thrombocytopenia. However, it does not alter platelet transfusion needs in the setting of high-dose chemotherapy and autologous bone marrow transplantation (13), and its narrow therapeutic window and side-effect profile limit its use. New TPO mimetics (14), some FDA approved to treat immune thrombocytopenic purpura, have rekindled hope that imaginative use of TPO receptor agonists will provide meaningful new approaches to treat a broad range of thrombocytopenic conditions, but this remains to be proven.

Beyond biomolecules, others have focused on developing novel cellular therapies for thrombocytopenia. New methods to generate megakaryocytes (15) and platelets (16) from human embryonic stem cells raise the possibility of a potentially endless platelet supply, but this approach has tremendous technical and safety hurdles before it could reach the clinic. More realistically, TPO is being explored ex vivo as a way to expand megakaryocyte progenitors in cord blood samples to hasten time to platelet independence (17). 


\section{Expanding demand for platelets}

The latest published report of blood product usage in the United States provides data only through 2001 (18). More current annual data from the state of California show that platelet usage has increased every year from 2000 through 2009, increasing roughly $70 \%$ over this time period without any sign of leveling off (K.-A. Nguyen, Blood Centers of the Pacific, San Francisco, California, USA, personal communication). The National Marrow Donor Program predicts a doubling in allogeneic transplants between 2010 and 2015, which is certain to tax platelet supplies. It is therefore imperative that we seek novel treatments for thrombocytopenia, especially therapyrelated thrombocytopenia, a major setting for prophylactic platelet transfusions and platelet refractoriness - which brings us to the report by Fuentes and colleagues in this issue of the JCI (19). Given that megakaryocytes have been found in the pulmonary vasculature, where some suggest that they release platelets (20), Fuentes et al. tested the hypothesis that infused murine megakaryocytes could yield quantitatively useful and biologically functional platelets in mouse recipients (19).

\section{Transfused megakaryocytes yield functioning circulating platelets}

Fuentes and colleagues used megakaryocytes derived from mouse fetal liver (19). In brief, harvested cells were cultured to expand and mature megakaryocyte lineage cells, a TPO-dependent process in which most cells in the dish express the megakaryocyte marker CD41 by the end of the culture period. Gravity sedimentation separation yielded two populations of cells: (a) larger, high-ploidy megakaryocytes and (b) smaller, low-ploidy cells. Intravenous transfusions were performed using mice that allowed for easy distinction between donor and recipient platelets based on antigenic differences in the platelet integrin $\alpha I I b$.

Each cell fraction was transfused independently, and mouse platelets were transfused as positive controls (19). Platelets yielded a peak platelet count 5 minutes after infusion, with a half-life of 36 hours, compared with a peak platelet count at 90 minutes and a 20-hour half-life for infused large megakaryocytes. Infused small cells, which contained a high number of cell-free proplatelets, yielded a peak platelet count at 5 minutes, similar to infused platelets, but with a circulating half-life of only 2 hours. Similar results were obtained using mar- row-derived megakaryocytes. These findings suggest that platelet release occurs predominantly from large, mature megakaryocytes, as postulated by Wright in 1910 (2). To simulate a myelosuppressed state, infusions were performed 7 days after highdose radiation, with platelet counts roughly $10 \%-20 \%$ of normal (19). Infusion of the large megakaryocyte cell fraction markedly increased the platelet count above controls, and the effect lasted more than 24 hours, closely mirroring platelet transfusions.

Two in vivo assays were used by Fuentes et al. to characterize the hemostatic function of the megakaryocyte-derived platelets (19). Using a laser injury model to visualize in situ clot formation, infused platelets and platelets derived from infused large megakaryocytes were shown to incorporate similarly into developing hemostatic platelet plugs. The megakaryocyte-derived platelets also functioned similarly to infused platelets in a $\mathrm{FeCl}_{3}$ carotid artery injury model that measures time to vascular occlusion, further indicating that they have hemostatic activity. In a provocative experiment, Fuentes and colleagues demonstrated that platelets shed from infused urokinase-expressing megakaryocytes delivered bioactive urokinase to a developing platelet plug, raising the possibility that infused megakaryocytes could be used to deliver desirable biological molecules to sites of vascular injury.

To understand where platelets are released following megakaryocyte infusion, mice were sacrificed at various time points following infusion of BrdU-labeled megakaryocytes (19). Nearly all BrdU ${ }^{+}$cells were found in the lungs, with very few in the spleen and none detected in the liver, heart, brain, or bone marrow. While suggestive, this finding does not prove that the lung is the site of platelet release, nor does it help us understand the role of the pulmonary vasculature in the normal physiology of platelet release. Is there something unique about pulmonary vasculature that makes it an ideal site for platelet release, or is it simply that the pulmonary capillary bed provides the first narrow passageway that cannot be traversed by infused large megakaryocytes? Comparing platelet count recovery following intravenous and intra-arterial megakaryocyte infusion will be informative.

\section{Future directions}

In this issue of the JCI, Fuentes and colleagues provide preliminary proof of principle for a new approach to treat thrombocytopenia (19), but now the hard work begins. They calculate that it will take roughly $10^{9}$ large megakaryocytes to achieve a $10 \%$ rise in platelet count in an average $70-\mathrm{kg}$ patient (19). They also estimate an average of 100-200 platelets released per infused megakaryocyte, so $10^{9}$ megakaryocytes would yield $1 \times 10^{11}$ to $2 \times 10^{11}$ platelets. To put this into perspective, platelet apheresis units, by definition, contain $3 \times 10^{11}$ to $6 \times 10^{11}$ platelets; so $10^{9}$ megakaryocytes might be a low estimate for the numbers needed in the clinical arena.

Although Fuentes et al. showed efficacy for bone marrow- and fetal liver-derived megakaryocytes, most of their data came from fetal liver cell preparations (19). The first order of business is to demonstrate that human megakaryocytes yield similar results. Once this is confirmed, what cell source should be used to apply this clinically? Should it be a human cell line, one that is genetically modified to enhance the efficiency of developing large megakaryocytes? Could one use cord blood cell units that contain inadequate cell numbers even for double cord transplants, of which there are many available in storage? In addition, issues of efficacy across HLA barriers and utility in patients who are refractory to standard platelet transfusions, as well as many others, need to be resolved. Moreover, safety, including a possible concern for transfusion-associated lung injury, will have to be addressed. However, the report by Fuentes et al. (19) pushes open an important therapeutic door by validating a potential new cell-based treatment for thrombocytopenia.

\section{Acknowledgments}

Andrew D. Leavitt's research efforts are funded by the California Institute for Regenerative Medicine (CIRM) and the NIH.

Address correspondence to: Andrew D. Leavitt, 513 Parnassus Ave., Room S-561, Box 0100, University of California, San Francisco, San Francisco, California, 941430100, USA. Phone: 415.514.3432; Fax: 415.514.3433; E-mail: leavitta@labmed2. ucsf.edu.

1. Wright JH. A rapid method for the differential staining of blood films and malarial parasites. J Med Res. 1902;7(1):138-144.

2. Wright JH. The histogenesis of the blood platelets. J Morphol. 1910;21(2):263-278.

3. Italiano JE Jr, Patel-Hett S, Hartwig JH. Mechanics of proplatelet elaboration. J Thromb Haemost. 2007;5(suppl 1):18-23.

4. Schwertz H, et al. Anucleate platelets generate progeny. Blood. 2010;115(18):3801-3809.

5. de Sauvage FJ, et al. Stimulation of megakaryocytopoiesis and thrombopoiesis by the $\mathrm{c}-\mathrm{Mpl}$ ligand. 
Nature. 1994;369(6481):533-538.

6. Kaushansky K, et al. Promotion of megakaryocyte progenitor expansion and differentiation by the c-Mpl ligand thrombopoietin. Nature. 1994;369(6481):568-571.

7. Lok S, et al. Cloning and expression of murine thrombopoietin cDNA and stimulation of platelet production in vivo. Nature. 1994;369(6481):565-568.

8. Kuter DJ, Begley CG. Recombinant human thrombopoietin: basic biology and evaluation of clinical studies. Blood. 2002;100(10):3457-3469.

9. Vadhan-Raj S, et al. Recombinant human thrombopoietin attenuates carboplatin-induced severe thrombocytopenia and the need for platelet transfusions in patients with gynecologic cancer. Ann Intern Med. 2000;132(5):364-368.

10. Fibbe WE, et al. Accelerated reconstitution of platelets and erythrocytes after syngeneic transplantation of bone marrow cells derived from thrombopoietin pretreated donor mice. Blood. 1995;86(9):3308-3313.
11. Somlo G, et al. Recombinant human thrombopoietin in combination with granulocyte colony-stimulating factor enhances mobilization of peripheral blood progenitor cells, increases peripheral blood platelet concentration, and accelerates hematopoietic recovery following high-dose chemotherapy. Blood. 1999;93(9):2798-2806.

12. Kuter DJ, et al. Thrombopoietin therapy increases platelet yields in healthy platelet donors. Blood. 2001;98(5):1339-1345

13. Vredenburgh JJ, et al. A randomized trial of recombinant human interleukin-11 following autologous bone marrow transplantation with peripheral blood progenitor cell support in patients with breast cancer. Biol Blood Marrow Transplant. 1998;4(3):134-141.

14. Kuter DJ. Biology and chemistry of thrombopoietic agents. Semin Hematol. 2010;47(3):243-248.

15. Gaur M, Kamata T, Wang S, Moran B, Shattil SJ, Leavitt AD. Megakaryocytes derived from human embryonic stem cells: a genetically tractable system to study megakaryocytopoiesis and integrin function.
J Thromb Haemost. 2006;4(2):436-442.

16. Nishikii $\mathrm{H}$, et al. Metalloproteinase regulation improves in vitro generation of efficacious platelets from mouse embryonic stem cells. J Exp Med. 2008;205(8):1917-1927.

17. Chen TW, Hwang SM, Chu IM, Hsu SC, Hsieh TB, Yao CL. Characterization and transplantation of induced megakaryocytes from hematopoietic stem cells for rapid platelet recovery by a two-step serum-free procedure. Exp Hematol. 2009; 37(11):1330-1339.e5.

18. Sullivan MT, Cotten R, Read EJ, Wallace EL. Blood collection and transfusion in the United States in 2001. Transfusion. 2007;47(3):385-394.

19. Fuentes R, et al. Infusion of mature megakaryocytes into mice yields functional platelets. J Clin Invest. 2010;120(11):3917-3922.

20. Zucker-Franklin D, Philipp CS. Platelet production in the pulmonary capillary bed: new ultrastructural evidence for an old concept. Am J Pathol. 2000;157(1):69-74.

\section{New therapy to revert dysfunctional antibody responses during HIV-1 infection}

Francesca Chiodi

Department of Microbiology, Tumor and Cell Biology, Karolinska Institutet, Stockholm, Sweden.

\begin{abstract}
Individuals infected with HIV-1 progress to AIDS at different rates. Rapid progressors develop AIDS within 2-5 years of initial infection, compared with approximately 10 years in typical progressors. Progression to AIDS is associated with impaired humoral and cellular immunity. In this issue of the JCI, Titanji and colleagues report that activated memory $B\left(\mathrm{mB}_{\mathrm{Act}}\right)$ cells are depleted in SIV-infected macaques defined as rapid progressors. Depletion was mediated by programmed death-1 (PD-1) and resulted in reduction of antibody titers specific for SIV and bacterial antigens. Interestingly, blockade of PD-1 in infected animals protected B cells from apoptosis and increased levels of SIV-specific antibodies in blood. These findings pave the way for a new therapeutic strategy aimed at improving humoral immunity in HIV-1 infection.
\end{abstract}

The use of antiretroviral therapy (ART) has dramatically reduced the number of individuals infected with HIV-1 that rapidly progress to AIDS in developed countries. However, rapid progression remains a problem in developing countries, where the majority of individuals infected with HIV-1 live, because use of ART is limited. Patients are clinically determined to have progressed to AIDS when their $\mathrm{CD}^{+} \mathrm{T}$ cell counts drop below 200 cells per microliter of blood. Although $\mathrm{CD}^{+}$ $\mathrm{T}$ cell counts remain the gold standard to predict immunological impairment

Conflict of interest: F. Chiodi is a founder of and shareholder in Imed AB, a start-up company at the Karolinska Institutet Science Park.

Citation for this article: $J$ Clin Invest. 2010; 120(11):3810-3813. doi:10.1172/JCI44872. upon disease progression and to monitor immunological recovery during ART, the quality of responses mediated by $\mathrm{CD}^{+}$ T cells during HIV-1 infection does not always correlate with their numbers (1). Thus, the identification of immunological biomarkers that reflect pathogenic events occurring during HIV-1 infection and that are predictive of progression to AIDS is an important research goal.

Progression to AIDS is associated with increased susceptibility to opportunistic infections as a result of both impaired cellular immune responses and dysfunctional humoral responses. In this regard, loss of memory B cells has been shown to lead to impaired HIV-specific and non-HIV-specific humoral immune responses (2). Understanding more clearly the mechanisms underlying $B$ cell deple- tion during HIV-1 infection and the role of $\mathrm{B}$ cell defects in disease pathogenesis and progression could prove informative for developing approaches to improve humoral immunity in patients infected with HIV-1.

\section{Depletion of memory B cells during SIV infection}

In this issue of the JCI, Titanji and colleagues present new results on depletion of activated memory $B\left(\mathrm{mB}_{\text {Act }}\right)$ cells during the early phases of SIV infection in rhesus macaques rapidly progressing to AIDS (3). In their study, depletion of this B cell subset was found to be associated with failure to produce SIV-specific antibodies. Alteration in B cell phenotype and in the percentages of different $B$ cell subsets has also been described during acute and chronic phases of HIV-1 infection, with depletion of memory B cells (4-6). Accordingly, it is likely that the impaired capacity to mount an efficient neutralizing antibody response to HIV-1 may be directly correlated to the damage occurring to B cells devoted to antibody production.

SIV infection in macaques defined as rapid progressors not only affected SIVspecific memory B cells, but randomly eradicated memory B cells specific for other previously encountered antigens. 